\section{Liquor-Cystatin C}

T. O. Kleine

Institut für Laboratoriumsmedizin und Pathobiochemie, Molekulare Diagnostik Standort Marburg Referenzlabor für Liquordiagnostik, UKGM Universitätsklinikum Gießen und Marburg, Marburg, Deutschland

Synonym(e) Gamma-trace; Post- $\gamma$-Protein; Post- $\gamma$-Globulin

Englischer Begriff cystatin C in cerebrospinal fluid (CSF)

Definition Humanes Cystatin C (Cys C; $13 \mathrm{kDa}$ ) ist ein Typ-2-Cys-Protease-Inhibitor mit 120 Aminosäuren, 2 Disulfidgruppen und ca. 9 verschiedenen Glycoformen, basisch detektiert mittels isoelektrischer Fokussierung und Silberfärbung ( $\triangleright$ Liquor-Banden, oligoklonale; $\triangleright$ Cystatin C).
Beschreibung In CSF und Blut-Plasma von Erwachsenen zeigt ein CSF/Plasma-Quotient von 2,3-14,6 höhere Cys-CKonzentrationen [Cys C] in CSF von 3,2-12,5 mg/L an und niedrigere $[$ Cys $\mathrm{C}$ ] in Plasma von $0,63-2,25 \mathrm{mg} / \mathrm{L}$, was eine Cys-C-Bildung im Zentralnervensystem indiziert: Kulturen von Choroid-Plexus, Neuronen und leptomeningealen Zellen von Nagetieren synthetisieren Cys $\mathrm{C}$ in vitro.

\section{Literatur}

Ghidoni R, Paterlini A, Albertini V et al (2011) Cystatin C is released in association with exosomes: a new tool of neuronal communication which is unbalanced in Alzheimer's disease. Neurobiol Aging 32:1435-1442

Löfberg H, Grubb AO (1979) Quantitation of $\gamma$-trace in human biological fluids: indications for production in the central nervous system. Scand J Clin Lab Invest 39:619-626

Ohe Y, Ishikawa K, Itoh Z et al (1996) Cultured leptomeningeal cells secrete cerebrospinal fluid proteins. J Neurochem 67:964-971 